\title{
Vibrational Activation of Methane Chemisorption: The Role of Symmetry
}

\author{
P. Morten Hundt, Maarten E. van Reijzen, Hirokazu Ueta, ${ }^{\ddagger}$ and Rainer D. Beck* \\ Laboratoire de Chimie Physique Moléculaire, Ecole Polytechnique Fédérale de Lausanne, 1015 Lausanne, Switzerland
}

ABSTRACT: Quantum state-resolved reactivity measurements probe the role of vibrational symmetry on the vibrational activation of the dissociative chemisorption of $\mathrm{CH}_{4}$ on $\mathrm{Pt}(111)$. IRIR double resonance excitation in a molecular beam is used to prepare $\mathrm{CH}_{4}$ in all three different vibrational symmetry components $\mathrm{A}_{1}, \mathrm{E}$, and $\mathrm{F}_{2}$ of the $2 \nu_{3}$ antisymmetric stretch overtone vibration. Methyl dissociation products chemisorbed on the cold $\mathrm{Pt}(111)$ surface are detected via reflection absorption infrared spectroscopy (RAIRS). We observe similar reactivity for $\mathrm{CH}_{4}$ prepared in the $A_{1}$ and $F_{2}$ sublevels but up to a factor of 2 lower reactivity for excitation of the $E$ sublevel. It is suggested that differences in the localization of the $\mathrm{C}-\mathrm{H}$ stretch amplitudes for the three states at the transition state leads to the observed difference in reactivity rather than statespecific vibrational energy transfer to electronic excitation of the metal.

SECTION: Surfaces, Interfaces, Porous Materials, and Catalysis

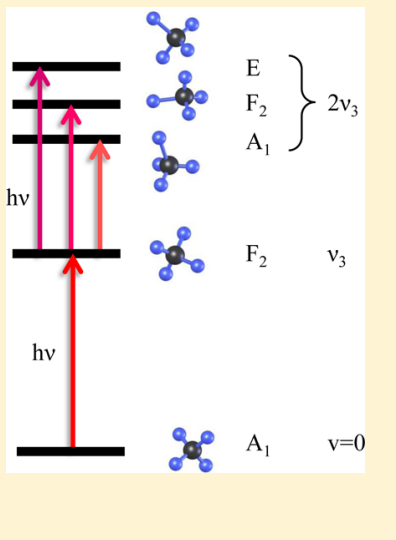

$S^{\text {then }}$ tate-resolved studies of methane dissociation on transitionmetal surfaces have been performed by several research groups to uncover the detailed dynamics of this prototype chemisorption reaction. ${ }^{1,2}$ Methane dissociation on metals is of particular interest due to its industrial applications in the steam reforming process, where the dissociation of methane on a catalyst surface is known to be the rate-limiting step $^{3}$ of the overall process. Another reason for the interest in this reaction is the fact that $\mathrm{CH}_{4}$ is a polyatomic molecule sufficiently complex to experimentally probe effects of mode specificity, ${ }^{4-7}$ bond selectivity, ${ }^{8,9}$ and steric effects ${ }^{10,11}$ and small enough to be tractable by high-level dynamical calculations. The highly detailed data obtained through state-resolved measurements provide stringent tests of theoretical models for gas/surface reactivity that are needed to develop a detailed understanding of this important class of chemical reactions.

The first evidence for mode specificity in a gas/surface reaction ${ }^{4}$ was obtained for the dissociation of $\mathrm{CH}_{2} \mathrm{D}_{2}$ on a $\mathrm{Ni}(100)$ surface. These experiments used direct overtone excitation to prepare the reactant $\mathrm{CH}_{2} \mathrm{D}_{2}$ in the $\mathrm{C}-\mathrm{H}$ stretch local mode states $|11\rangle$ and $|20\rangle$, which carry either one quantum of $\mathrm{C}-\mathrm{H}$ stretch in each of the two $\mathrm{C}-\mathrm{H}$ oscillators or two quanta of $\mathrm{C}-\mathrm{H}$ stretch in a single oscillator and none in the other, respectively. $\mathrm{CH}_{2} \mathrm{D}_{2}$ prepared in the $|20\rangle$ state was observed to be up to five times more reactive than that in the 111) state. The observed mode specificity was suggested to be due to the different localization of the $\mathrm{C}-\mathrm{H}$ stretch amplitude for the two reactant states $|20\rangle$ and $|11\rangle$. For the more reactive 120 ) state, the $\mathrm{C}-\mathrm{H}$ stretch amplitude is localized in a single $\mathrm{C}-\mathrm{H}$ bond that more closely resembles the transition state than the $|11\rangle$ state where the vibrational amplitude is equally distributed over the two $\mathrm{C}-\mathrm{H}$ bonds.
Another observation of mode specificity in methane chemisorption was reported by Maroni et al., ${ }^{6}$ who studied the dissociation of $\mathrm{CH}_{4}\left(\nu_{1}\right)$ on $\mathrm{Ni}(100)$, prepared by stimulated Raman pumping in the totally symmetric $\left(A_{1}\right)$ $\mathrm{C}-\mathrm{H}$ stretch normal mode $\nu_{1}$. Their results demonstrated that the $\nu_{1}$ vibration is much more efficient in promoting the reaction than the isoenergetic antisymmetric $\mathrm{C}-\mathrm{H}$ stretch mode $\nu_{3}$ of $\mathrm{F}_{2}$ symmetry that was studied by Juurlink et al. ${ }^{12}$ The difference in reactivity between $\mathrm{CH}_{4}\left(\nu_{1}\right)$ and $\mathrm{CH}_{4}\left(\nu_{3}\right)$ cannot be explained in terms of different localization of the vibrational amplitude for the initial vibrational states $\nu_{1}$ and $\nu_{3}$ because for both states, all four $\mathrm{C}-\mathrm{H}$ bonds carry the same amplitude and differ only in their relative phase. Halonen et al. $^{13}$ described a four-dimensional vibrationally adiabatic model of the evolution of the $\mathrm{CH}_{4}$ stretching states during methane's approach to the surface. Their model suggests higher reactivity for $\mathrm{CH}_{4}$ initially prepared in the $\nu_{1}$ normal mode rather than that in the nearly isoenergetic $\nu_{3}$ mode due to different intramolecular energy flow for the two modes resulting from the molecule/surface interaction during the approach of reactant to surface. This surface-induced intramolecular vibrational redistribution (IVR) process $^{14}$ leads to different localization of the $\mathrm{C}-\mathrm{H}$ amplitudes with the $\nu_{1}$ mode evolving toward a stretch excitation of the $\mathrm{C}-\mathrm{H}$ bond closest to the surface, whereas the $\nu_{3}$ mode results in $\mathrm{C}-\mathrm{H}$ stretch amplitude in the distal $\mathrm{CH}_{3}$ group.

More recently, the group of Bret Jackson developed a theoretical model for $\mathrm{CH}_{4}$ chemisorption based on a reaction path Hamiltonian approach that includes all 15 internal degrees

Received: April 11, 2014

Accepted: May 16, 2014 
of freedom of the incident $\mathrm{CH}_{4}{ }^{15,16}$ This model again predicts a higher reactivity for $\mathrm{CH}_{4}\left(\nu_{1}\right)$ compared to $\mathrm{CH}_{4}\left(\nu_{3}\right)$ due to strong vibrationally nonadiabatic coupling to the ground state and significant mode softening at the transition state.

An alternative explanation for the mode-specific reactivity of $\mathrm{CH}_{4}\left(\nu_{1}\right)$ and $\mathrm{CH}_{4}\left(\nu_{3}\right)$ invokes the difference in vibrational symmetry between the two states. While the totally symmetric and infrared inactive $\nu_{1}$ vibration carries no transition dipole moment from the ground state, the antisymmetric $\nu_{3}$ vibration is infrared-active with a nonzero transition dipole moment. Classically, $\mathrm{CH}_{4}\left(\nu_{3}\right)$ corresponds to an oscillating electric dipole approaching a conducting surface where an opposite image dipole will be induced in the metal surface. Dipoledipole coupling could lead to vibrational energy transfer between the incident vibrationally excited molecules and electronic excitations of the metal. ${ }^{17,18}$ The infrared-active $\nu_{3}$ vibration of $\mathrm{F}_{2}$ symmetry could therefore be damped by such an image dipole mechanism opening a possible de-excitation pathway, which could account for the lower $\mathrm{CH}_{4}\left(\nu_{3}\right)$ reactivity. On the other hand, the infrared-inactive $\nu_{1}$ vibration of $\mathrm{A}_{1}$ symmetry cannot transfer energy via this dipole-dipole mechanism and will be unaffected. While there is currently no evidence that methane chemisorption involves electronically nonadiabatic processes, such a mechanism is conceivable and would be consistent with the observed mode specificity for the $\nu_{1}$ and $\nu_{3}$ vibrations.

The first overtone vibration $2 \nu_{3}$ of the triply degenerate $\nu_{3}$ vibration offers a further possibility to probe the effect of vibrational symmetry on the $\mathrm{CH}_{4}$ reactivity. While the $2 \nu_{3}$ overtone vibration is six-fold degenerate in the harmonic approximation, it splits due to anharmonicity into three vibrational components with $\mathrm{A}_{1}, \mathrm{E}$, and $\mathrm{F}_{2}$ symmetry in the $T_{d}$ group. Of these, only the $\mathrm{F}_{2}$ component is accessible by direct single-photon overtone excitation from the $v=0$ ground state of $A_{1}$ symmetry. However, using IR-IR double resonance excitation with two infrared photons, it is possible to prepare $\mathrm{CH}_{4}\left(2 \nu_{3}\right)$ in all three vibrational symmetry components ${ }^{19,20}$ and compare the effect of these nearly isoenergetic vibrations on the dissociative chemisorption probabilities of $\mathrm{CH}_{4}$ on a clean $\mathrm{Pt}(111)$ surface. A simplified energy level diagram of the excitation path is shown in Figure 1.

The molecular beam/surface science apparatus, state-specific reactant preparation by rapid adiabatic passage (RAP), and product detection by reflection absorption infrared spectroscopy (RAIRS) have been described in previous publications. ${ }^{21-23}$ Briefly, a molecular beam of methane is generated by expanding pure methane or $3 \% \mathrm{CH}_{4}$ seeded in He with a backing pressure of 3 bar through a nozzle of $50 \mu \mathrm{m}$ diameter. A nickel skimmer of $1 \mathrm{~mm}$ diameter extracts a molecular beam from this supersonic expansion. Molecular beam speed distributions as a function of seed ratio and nozzle temperature were determined by measuring the molecules' time-of-flight with an on-axis quadrupole mass spectrometer (QMS) in combination with a fast chopper wheel. ${ }^{21}$ In order to determine reactive sticking coefficients of $\mathrm{CH}_{4}$, the flux of methane incident on the $\mathrm{Pt}(111)$ surface was monitored by a calibrated QMS located in the UHV chamber, and the surface coverage of adsorbed methyl products was detected by RAIRS during the deposition.

Rovibrational excitation of methane in the molecular beam was achieved using two continuous infrared optical parametric oscillators (OPOs). The OPO idler outputs were frequency stabilized to two sequential rovibrational transitions $(\nu=0$ to 1 to 2) of $\mathrm{CH}_{4}$ using Doppler-free absorption features in static

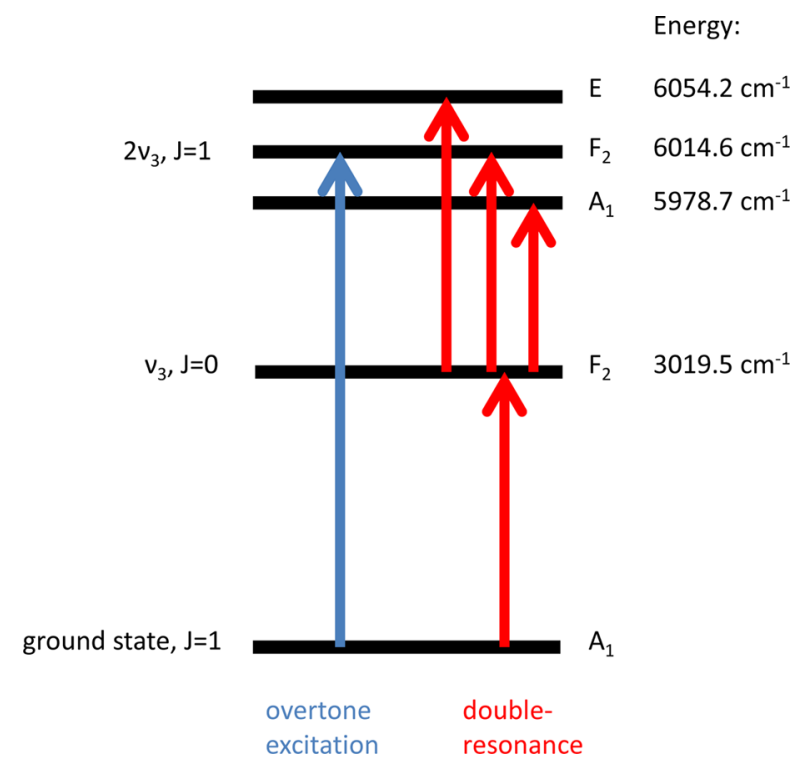

Figure 1. Energy level diagram of $\mathrm{CH}_{4}$ antisymmetric $\mathrm{C}-\mathrm{H}$ stretch vibrations $\nu_{3}$ and $2 \nu_{3}$. The $2 \nu_{3}$ vibration is split into three vibrational symmetry species $\left(E, F_{2}\right.$, and $\left.A_{1}\right)$ due to molecular symmetry splitting. Only the $2 \nu_{3}-\mathrm{F}_{2}$ component is infrared-active, that is, accessible by a single-photon transition from the ground state. However, double resonance excitation via the $\nu_{3}$ state allows the excitation of all three vibrational symmetry components of $2 \nu_{3}$.

gas cells. OPO1 was stabilized to a Lamb dip of the $\mathrm{P}(1) \nu_{3}$ transition of $\mathrm{CH}_{4}$ detected in the Doppler-broadened absorption line using a static gas cell at room temperature ${ }^{24}$ (Figure 2a). The output of OPO1 traversed a second gas cell of $90 \mathrm{~cm}$ length filled with $150 \mu$ bar of $\mathrm{CH}_{4}$, where it selectively created population of the $\nu_{3}=1, J=0$ state for molecules moving perpendicular to the IR beam. The Doppler-free population created by OPO1 was probed by overlapping the two IR beams in the gas cell and scanning OPO2 over the $\mathrm{R}(0)$, $\nu_{3}=2 \leftarrow \nu_{3}=1$ transition of E symmetry (Figure $2 \mathrm{~b}$ ). OPO2 was then frequency locked to the peak of the Doppler-free absorption signal with a FWHM of $3.5 \mathrm{MHz}$.

The infrared beams of the frequency-stabilized OPOs crossed the molecular beam in two locations separated by $30 \mathrm{~mm}$ to excite $\mathrm{CH}_{4}$ in an IR-IR double resonance scheme using RAP $275 \mathrm{~mm}$ from the $\mathrm{Pt}(111)$ surface. Details of the RAP excitation have been described previously. ${ }^{23}$ A pyroelectric detector that could be moved into the molecular beam was used to detect and quantify the flux of vibrationally excited molecules in the molecular beam and to verify that complete population inversion was achieved for both excitation steps.

RAIRS was used to monitor the uptake of chemisorbed methyl species, $\mathrm{CH}_{3}(\mathrm{ads})$, on the $\mathrm{Pt}(111)$ surface formed as a product of the dissociative chemisorption of $\mathrm{CH}_{4}$ at a surface temperature of $150 \mathrm{~K}$. Comparison of the RAIRS absorption signal for the symmetric $\mathrm{CH}_{3}$ (ads) stretch mode at $2883 \mathrm{~cm}^{-1}$ with Auger detection of the carbon signal on $\mathrm{Pt}(111)$ was used to verify the linear relation between RAIRS peak absorption signal and methyl coverage and to calibrate the RAIRS peak signal in terms of methyl coverage. ${ }^{21}$

Double resonance excitation in combination with RAIRS detection was used to measure the state-resolved dissociation probability of $\mathrm{CH}_{4}$ on $\mathrm{Pt}(111)$ for three symmetry components of the $2 \nu_{3}$ overtone states. Figure 3 shows RAIRS-detected uptake curves that relate the $\mathrm{CH}_{3}$ (ads) coverage observed by 

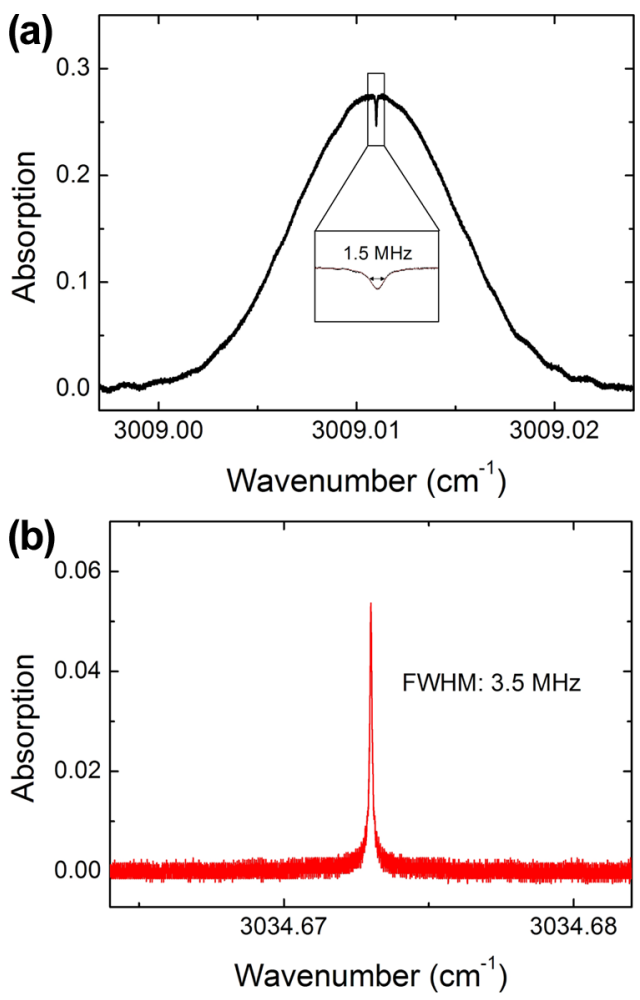

Figure 2. (a) Lamb dip of the $\mathrm{P}(1)-\nu_{3}$ transition of $\mathrm{CH}_{4}$ detected in a $1.6 \mathrm{~m}$ long gas cell at a pressure of $60 \mu$ bar. (b) Doppler-free absorption of the $\mathrm{R}(0), \nu_{3}=2 \leftarrow \nu_{3}=1$ transition of E symmetry in a static gas cell of $90 \mathrm{~cm}$ length filled with $150 \mu$ bar of $\mathrm{CH}_{4}$. The population in $\nu_{3}$ is created by pumping with OPO1.

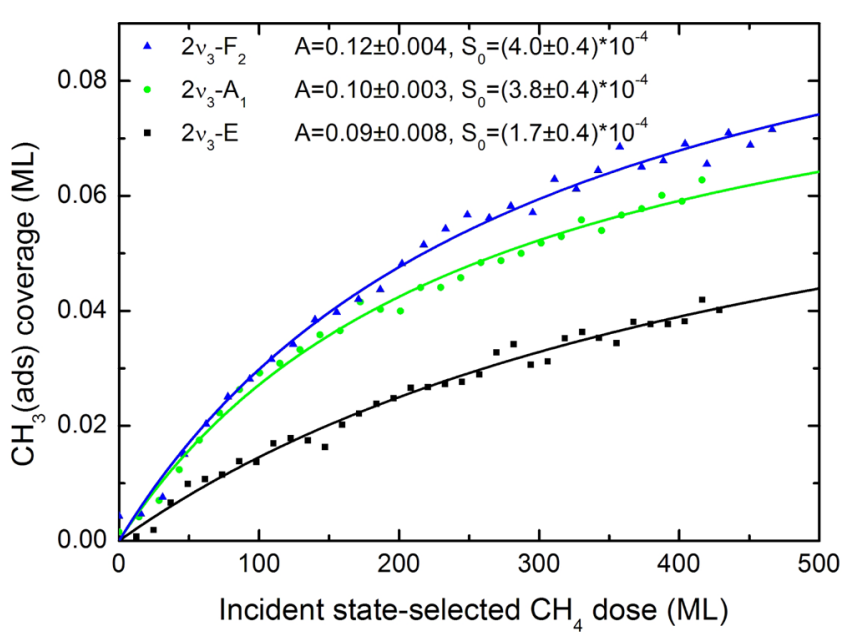

Figure 3. RAIRS-detected uptake curves showing $\mathrm{CH}_{3}$ (ads) coverage on $\mathrm{Pt}(111)$ at $T_{s}=150 \mathrm{~K}$ as a function of incident dose of stateprepared $\mathrm{CH}_{4}$ for each of the symmetry components $\mathrm{A}_{1}, \mathrm{E}$, and $\mathrm{F}_{2}$ of the $2 \nu_{3}$ vibration. The average incident translational energy was $E_{\mathrm{t}}=$ $12.8 \mathrm{~kJ} / \mathrm{mol}$ using a $100 \% \mathrm{CH}_{4}$ expansion with a nozzle temperature $T_{\mathrm{n}}=400 \mathrm{~K}$. The initial slopes of the uptake curves yield the stateresolved initial sticking probability $S_{0}$, and the asymptote A corresponds to the saturation coverage.

RAIRS to the incident $\mathrm{CH}_{4}$ dose measured by the pyroelectric detector. We note that under the conditions used in our experiments, the observed $\mathrm{CH}_{3}$ (ads) uptake is exclusively due to dissociation of the overtone excited $\mathrm{CH}_{4}\left(2 \nu_{3}\right)$. Any $\mathrm{CH}_{4}(v=$ $0)$ present in the beam has insufficient energy to surmount the reaction barrier and therefore does not contribute to the observed $\mathrm{CH}_{3}$ (ads) uptake. The slope of the measured uptake curve corresponds to the coverage-dependent sticking probability $S(\theta)$. We determine the initial sticking coefficient $S_{0}$ by fitting a Langmuir uptake model ${ }^{25}$ of the form

$$
\theta(D)=A \cdot \frac{B \cdot D}{1+B \cdot D}
$$

where $D$ is the incident $\mathrm{CH}_{4}$ dose (in monolayer, $\mathrm{ML}$ ), $A$ is the saturation coverage, and $B$ is a fitting constant. The initial sticking coefficient $S_{0}$ corresponds the initial slope of the uptake curve, which is given by the product $A \cdot B$. Uptake curves for $\mathrm{CH}_{4}$ prepared in the three states of different vibrational symmetries are presented in Figure 3. The uptake curves obtained for preparation of the incident $\mathrm{CH}_{4}$ in the $2 \nu_{3}-\mathrm{A}_{1}$ and $2 \nu_{3}-\mathrm{F}_{2}$ states show a steeper initial rise than for the $2 \nu_{3}-\mathrm{E}$ state. This indicates a lower reactivity for the nearly isoenergetic state of $\mathrm{E}$ symmetry compared to the states of $\mathrm{A}_{1}$ and $\mathrm{F}_{2}$ symmetry. Besides the difference in the initial slope, the uptake curves also approach different asymptotes at high incident dose. This indicates that the $\mathrm{CH}_{3}$ (ads) saturation coverage on the $\mathrm{Pt}(111)$ surface depends both on the rovibrational quantum state and on the translational energy of the incident methane molecules. Recent work from our group published jointly with theory by the group of Bret Jackson showed that the saturation coverage of $\mathrm{CH}_{3}$ on a $\mathrm{Pt}(111)$ surface increases with increasing reactivity due to a coverage dependence of the reaction barrier height. $^{22}$ At constant reactivity of the incident methane molecules, the $\mathrm{CH}_{3}$ (ads) uptake will saturate at a certain coverage if the incident molecules are no longer able to overcome the reaction barrier. An increase in reactivity of the incident $\mathrm{CH}_{4}$, either due to an increase in translational energy or excitation to a more reactive state, will cause a continued uptake of $\mathrm{CH}_{3}$, leading to a higher apparent saturation coverage. This effect was also observed here. We observe the highest saturation coverage for the state with the largest initial sticking coefficients.

Figure 4 shows the state-resolved initial sticking coefficients $S_{0}$ obtained from the initial slope of the uptake curves for the

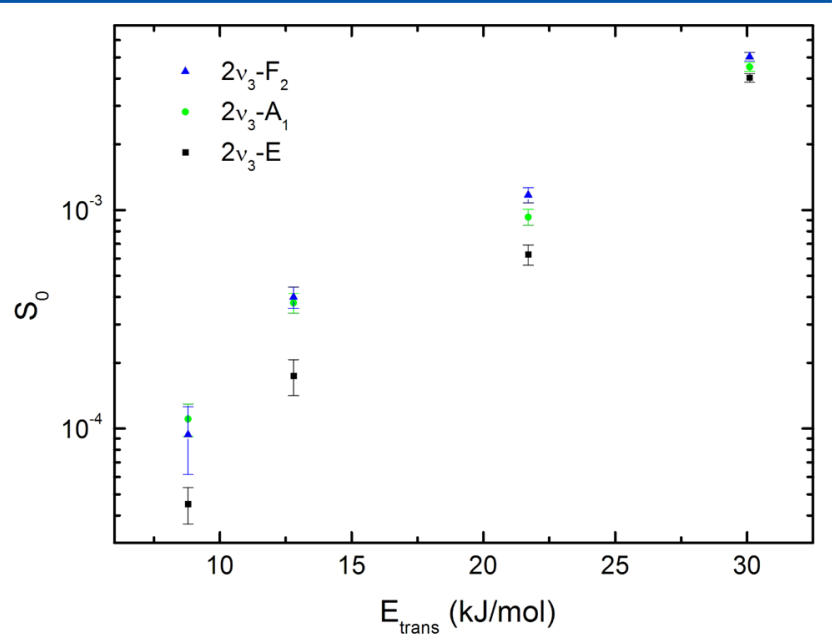

Figure 4. State-resolved initial dissociative sticking coefficients $S_{0}$ of $\mathrm{CH}_{4}$ on $\mathrm{Pt}(111)$ for the three symmetry species $\mathrm{A}_{1}, \mathrm{E}$, and $\mathrm{F}_{2}$ as a function of average incident translational energy $E_{\mathrm{t}}$ and $T_{\mathrm{s}}=150 \mathrm{~K} . E_{\mathrm{t}}$ was varied by changing the seed ratio of the expansion gas $\left(100 \% \mathrm{CH}_{4}\right.$ and $3 \% \mathrm{CH}_{4}$ in $\left.\mathrm{He}\right)$ and the nozzle temperature $\left(T_{\mathrm{n}}=300\right.$ and 400 $\mathrm{K})$. 
three $2 \nu_{3}$ states of different vibrational symmetry as a function of incident translational energy $E_{\mathrm{t}}$. The data shows a nearexponential increase in reactivity with increasing $E_{\mathrm{t}}$. Over the $E_{\mathrm{t}}$ range explored here, the $2 \nu_{3}-\mathrm{A}_{1}$ and $2 \nu_{3}-\mathrm{F}_{2}$ states show higher reactivity than the $2 \nu_{3}-\mathrm{E}$ state with a decreasing difference in reactivity as $E_{\mathrm{t}}$ increases.

A difference in reactivity between nearly isoenergetic states of different vibrational symmetry demonstrates the presence of mode specificity in chemisorption of $\mathrm{CH}_{4}$ on $\mathrm{Pt}(111)$. Mode specificity occurs when the reactivity is not only a function of the total internal vibrational energy of the incident reactant molecule but depends on the specific rovibrational quantum state. The observation that $\mathrm{CH}_{4}$ prepared in the totally symmetric $2 \nu_{3}-\mathrm{A}_{1}$ state is no more reactive than $\mathrm{CH}_{4}\left(2 \nu_{3}-\mathrm{F}_{2}\right)$ may seem surprising in light of the strong difference in reactivity between $\mathrm{CH}_{4}\left(\nu_{1}-\mathrm{A}_{1}\right)$ and $\mathrm{CH}_{4}\left(\nu_{3}-\mathrm{F}_{2}\right)$ previously observed on $\mathrm{Ni}(100)$. It indicates that $\mathrm{CH}_{4}$ prepared in a totally symmetric vibrational state is not always more reactive than that for preparation in a state that carries a transition dipole moment to the vibrational ground state. This result suggests that the mode specificity observed here for the $2 \nu_{3}$ states of different symmetry and previously for the normal modes $\nu_{1}$ and $\nu_{3}^{6,12}$ is not due to different de-excitation rates by vibrational energy transfer from the incident molecules to electronic excitation of the metal via an oscillating dipole-image dipole coupling. $^{17,18}$

Rather, the mode specificity observed for the $2 \nu_{3}$ states of $F_{2}$ and $A_{1}$ symmetry and the $2 \nu_{3}-\mathrm{E}$ state appears to result from different localization of the $\mathrm{C}-\mathrm{H}$ stretch amplitudes at the transition state for the states of different symmetry. Abram et $\mathrm{al}^{26}$ analyzed the $\mathrm{C}-\mathrm{H}$ stretch overtone states of $\mathrm{CH}_{4}$ using anharmonic symmetrized internal coordinates (SICs). They showed that for states containing two quanta of $\mathrm{C}-\mathrm{H}$ stretch, the $\mathrm{C}-\mathrm{H}$ stretch amplitudes are partially localized in a single $\mathrm{C}-\mathrm{H}$ bond for the $\mathrm{A}_{1}$ and $\mathrm{F}_{2}$ symmetry states, that is, the wave functions contain some $|2000\rangle$ local mode character, while for the $\mathrm{E}$ symmetry state, the $\mathrm{C}-\mathrm{H}$ amplitude is completely delocalized and contains only $|1100\rangle$ character.

More recent calculations by Halonen ${ }^{27}$ using a local mode basis predict similar $\mathrm{C}-\mathrm{H}$ localization for the $2 \nu_{3}$ overtone states of $\mathrm{CH}_{4}$. Alternatively, the eigenstates of methane can be described in a normal mode basis $\left(\nu_{1}, \nu_{2}, \nu_{3}, \nu_{4}\right)$. Venuti et al. ${ }^{28}$ and Wang et al. ${ }^{29}$ calculated the vibrational frequencies of methane up to $9000 \mathrm{~cm}^{-1}$ and give the leading terms for a normal mode expansion of the eigenstates. Their analysis shows that of the three $2 \nu_{3}$ symmetry components, only the $A_{1}$ and $F_{2}$ states contain a significant amount of $\nu_{1}$ character, whereas the least reactive $\mathrm{E}$ state contains no $\nu_{1}$ character.

The localization of the $\mathrm{C}-\mathrm{H}$ stretch amplitude in a single $\mathrm{C}-\mathrm{H}$ bond for the vibrations of $\mathrm{A}_{1}$ and $\mathrm{F}_{2}$ symmetry may be at least partly responsible for the higher reactivity of these states compared to the $\mathrm{E}$ state with its delocalized vibrational amplitude. Similar mode specificity has been observed previously for the dissociation of $\mathrm{CH}_{2} \mathrm{D}_{2}$ on $\mathrm{Ni}(100)$ described above, ${ }^{4}$ where the $|20\rangle$ local mode state was found to be more reactive than the delocalized $|11\rangle$ state. DFT calculations ${ }^{30}$ of the transition state structure for methane dissociation on $\mathrm{Pt}$ and $\mathrm{Ni}$ surfaces predict the dissociating $\mathrm{C}-\mathrm{H}$ bond to be significantly stretched $\left(r_{\mathrm{C}-\mathrm{H}}=1.49 \AA\right.$ for the transition state on $\mathrm{Pt}(111)$ compared to $1.09 \AA$ for the gas phase). Localizing the $\mathrm{C}-\mathrm{H}$ stretch amplitude in a single $\mathrm{C}-\mathrm{H}$ bond via excitation of a state with $|20\rangle$ character makes the reactant resemble the transition state more strongly than excitation of a $|11\rangle$ state, which distributes the $\mathrm{C}-\mathrm{H}$ amplitude over two $\mathrm{C}-\mathrm{H}$ bonds, one of which becomes a spectator in the dissociation. ${ }^{31}$ Jiang et $\mathrm{al}^{32}$ expressed this idea of deforming the reactant toward the transition-state structure in a quantitative way by proposing a sudden vector projection (SVP) model. The SVP model calculates the projection of the reactant vibrational state (vector) onto the transition-state structure and establishes a correlation between the magnitude of the SVP value for a given vibrational state and the promotional effect of this state on the reactivity. However, as a sudden model, the SVP model neglects the possibility of IVR that can occur during the approach of the reaction partners.

Killelea et al. ${ }^{14}$ recently discussed the role of surface-induced IVR in mode and bond selectivity for vibrationally mediated surface reactions. Also in cases where the laser-prepared vibrational state does not initially localize the $\mathrm{C}-\mathrm{H}$ stretch amplitude in a favorable way for the reaction, surface-induced IVR may cause energy flow within the reactant molecule and localize the vibrational amplitude at the transition state. ${ }^{13}$ Surface-induced IVR may therefore also contribute to the mode specificity observed for the $2 \nu_{3}$ states of $\mathrm{CH}_{4}$. Similarly, the mode specificity observed previously ${ }^{6,12}$ between the $\nu_{1}$ and $\nu_{3}$ normal modes was attributed to be due to surface-induced IVR by Halonen et al. ${ }^{13}$

More recently Jackson and co-workers ${ }^{15,16}$ developed a more detailed first-principles model for $\mathrm{CH}_{4}$ dissociation based on a minimum-energy reaction path calculated by density function theory and a harmonic potential for all 14 molecular degrees of freedom (including the 9 normal vibrational modes) orthogonal to the reaction path. Their fully quantum mechanical simulation uses wave packets propagating on vibrationally adiabatic potential energy surfaces, with nonadiabatic couplings linking the different vibrational states to each other. By design, their model includes surface-induced IVR leading to vibrational amplitude localization as well as mode softening at the transition state. Also included are vibrationally nonadiabatic couplings leading to energy flow between different vibrationally excited states and between excited vibrational states and the reaction path. Of all of the $\mathrm{CH}_{4}$ normal modes, the model predicts the $\nu_{1}$ mode to cause the largest increase in reactivity due to strong mode softening at the transition state as well as efficient conversion of the $\nu_{1}$ vibrational energy into kinetic energy along the ground-state reaction path, which at the transition state corresponds to breaking of the $\mathrm{C}-\mathrm{H}$ bond. Differences in mode softening and coupling to the ground state for the $2 \nu_{3}$ states of different symmetry may contribute to the mode specificity observed in our experiments. An extension of Jackson's reaction path Hamiltonian model to include states with more than one quantum of vibration will help to shed light on the detailed mechanism responsible for mode specificity of $2 \nu_{3}$ states of different symmetry reported here.

In summary, we have measured the state-resolved dissociation probability of $\mathrm{CH}_{4}$ on $\mathrm{Pt}(111)$ at $T_{\mathrm{s}}=150 \mathrm{~K}$ for the three different symmetry components $\mathrm{A}_{1}, \mathrm{E}$, and $\mathrm{F}_{2}$ of the $2 \nu_{3}$ vibration using double resonance excitation of the reactants and RAIRS detection of the $\mathrm{CH}_{3}$ (ads) products. Our results show that $\mathrm{CH}_{4}$ prepared in a totally symmetric $\mathrm{A}_{1}$ vibration is not necessarily more reactive than that in a vibrational state of lower symmetry such as $\mathrm{F}_{2}$ that carries a transition dipole moment to the vibrational ground state. We propose that the observed mode specificity for the three $2 \nu_{3}$ states is due to different $\mathrm{C}-\mathrm{H}$ stretch amplitude localization at the transition 
state that could either result directly from differences of the initially prepared state or be due to differences in the surfaceinduced IVR process. The state-resolved reactivity data obtained from our experiments can guide the development of detailed multidimensional dynamical models with the aim of arriving at a predictive understanding of this important gas/ surface reaction.

\section{AUTHOR INFORMATION}

\section{Corresponding Author}

*E-mail: rainer.beck@epfl.ch.

\section{Present Address}

${ }^{\ddagger}$ H.U.: Institute of Low Temperature Science, Hokkaido University, Sapporo 060-0819, Japan.

\section{Notes}

The authors declare no competing financial interest.

\section{ACKNOWLEDGMENTS}

We gratefully acknowledge financial support provided by the Swiss National Science Foundation (Grant No. 134709/1) and the Ecole Polytechnique Fédérale de Lausanne and helpful discussions with Bret Jackson.

\section{REFERENCES}

(1) Larsen, J. H.; Chorkendorff, I. From Fundamental Studies of Reactivity on Single Crystals to the Design of Catalysts. Surf. Sci. Rep. 1999, 35, 163-222.

(2) Juurlink, L. B. F.; Killelea, D. R.; Utz, A. L. State-Resolved Probes of Methane Dissociation Dynamics. Prog. Surf. Sci. 2009, 84, 69-134.

(3) Chorkendorff, I.; Niemantsverdriet, J. W. Concepts of Modern Catalysis and Kinetics; Wiley-VCH: New York, 2003.

(4) Beck, R. D.; Maroni, P.; Papageorgopoulos, D. C.; Dang, T. T.; Schmid, M. P.; Rizzo, T. R. Vibrational Mode-Specific Reaction of Methane on a Nickel Surface. Science 2003, 302, 98-100.

(5) Bisson, R.; Sacchi, M.; Beck, R. D. Mode-specific Reactivity of $\mathrm{CH} 4$ on $\mathrm{Pt}(110)-(1 \times 2)$ : The Concerted Role of Stretch and Bend Excitation. Phys. Rev. B 2010, 82, 121404.

(6) Maroni, P.; Papageorgopoulos, D. C.; Sacchi, M.; Dang, T. T.; Beck, R. D.; Rizzo, T. R. State-Resolved Gas-Surface Reactivity of Methane in the Symmetric C-H Stretch Vibration on Ni(100). Phys. Rev. Lett. 2005, 94, 246104.

(7) Juurlink, L. B. F.; Smith, R. R; Killelea, D. R.; Utz, A. L. Comparative Study of $\mathrm{C}-\mathrm{H}$ Stretch and Bend Vibrations in Methane Activation on $\mathrm{Ni}(100)$ and $\mathrm{Ni}(111)$. Phys. Rev. Lett. 2005, 94, 208303.

(8) Killelea, D. R.; Campbell, V. L.; Shuman, N. S.; Utz, A. L. BondSelective Control of a Heterogeneously Catalyzed Reaction. Science 2008, 319, 790-793.

(9) Chen, L.; Ueta, H.; Bisson, R.; Beck, R. D. Vibrationally BondSelected Chemisorption of Methane Isotopologues on $\operatorname{Pt}(111)$ Studied by Reflection Absorption Infrared Spectroscopy. Faraday Discuss. 2012, 157, 285-295.

(10) Yoder, B. L.; Bisson, R.; Beck, R. D. Steric Effects in the Chemisorption of Vibrationally Excited Methane on $\mathrm{Ni}(100)$. Science 2010, 329, 553-556.

(11) Yoder, B. L.; Bisson, R.; Hundt, P. M.; Beck, R. D. Alignment Dependent Chemisorption of Vibrationally Excited $\mathrm{CH}_{4}\left(\nu_{3}\right)$ on $\mathrm{Ni}(100), \mathrm{Ni}(110)$, and $\mathrm{Ni}(111)$. J. Chem. Phys. 2011, 135, 224703.

(12) Juurlink, L. B. F.; McCabe, P. R.; Smith, R. R.; DiCologero, C. L.; Utz, A. L. Eigenstate-Resolved Studies of Gas-Surface Reactivity: $\mathrm{CH}_{4}\left(\nu_{3}\right)$ Dissociation on $\mathrm{Ni}(100)$. Phys. Rev. Lett. 1999, 83, 868-871.

(13) Halonen, L.; Bernasek, S. L.; Nesbitt, D. J. Reactivity of Vibrationally Excited Methane on Nickel Surfaces. J. Chem. Phys. 2001, 115, 5611-5619.

(14) Killelea, D. R.; Utz, A. L. On the Origin of Mode- and BondSelectivity in Vibrationally Mediated Reactions on Surfaces. Phys. Chem. Chem. Phys. 2013, 15, 20545-20554.
(15) Nave, S.; Jackson, B. Vibrational Mode-Selective Chemistry: Methane Dissociation on Ni(100). Phys. Rev. B 2010, 81, 233408.

(16) Jackson, B.; Nave, S. The Dissociative Chemisorption of Methane on $\mathrm{Ni}(100)$ : Reaction Path Description of Mode-Selective Chemistry. J. Chem. Phys. 2011, 135, 114701.

(17) Persson, B. N. J.; Persson, M. Vibrational Lifetime for CO Adsorbed on $\mathrm{Cu}(100)$. Solid State Commun. 1980, 36, 175-179.

(18) Persson, B. N. J.; Persson, M. Damping of Vibrations in Molecules Adsorbed on a Metal-Surface. Surf. Sci. 1980, 97, 609-624.

(19) Demartino, A.; Frey, R.; Pradere, F. Observation of the $\nu_{3}-$ $2 \nu_{3}, \mathrm{~A} 1 \mathrm{~B}$ and of Methane. Chem. Phys. Lett. 1983, 100, 329-333.

(20) Demartino, A.; Frey, R.; Pradere, F. Double-Resonance Observation of the $2 \nu_{3}$, E State in Methane. Chem. Phys. Lett. 1983, 95, 200-204.

(21) Chen, L.; Ueta, H.; Bisson, R.; Beck, R. D. Quantum StateResolved Gas/Surface Reaction Dynamics Probed by Reflection Absorption Infrared Spectroscopy. Rev. Sci. Instrum. 2013, 84, 053902.

(22) Ueta, H.; Chen, L.; Beck, R. D.; Colon-Diaz, I.; Jackson, B. Quantum State-Resolved $\mathrm{CH}_{4}$ Dissociation on $\mathrm{Pt}(111)$ : Coverage Dependent Barrier Heights from Experiment and Density Functional Theory. Phys. Chem. Chem. Phys. 2013, 15, 20526-20535.

(23) Chadwick, H.; Hundt, P. M.; van Reijzen, M. E.; Yoder, B. L.; Beck, R. D. Quantum State Specific Reactant Preparation in a Molecular Beam by Rapid Adiabatic Passage. J. Chem. Phys. 2014, 140, 034321.

(24) Demtröder, W. Atoms, Molecules and Photons: An Introduction to Atomic-, Molecular- and Quantum-Physics; Springer: New York, 2010.

(25) Killelea, D. R.; Campbell, V. L.; Shuman, N. S.; Smith, R. R.; Utz, A. L. Surface Temperature Dependence of Methane Activation on Ni(111). J. Phys. Chem. C 2009, 113, 20618-20622.

(26) Abram, I.; Demartino, A.; Frey, R. Higher Excited Vibrational States of Polyatomic Molecules. J. Chem. Phys. 1982, 76, 5727-5738.

(27) Halonen, L. Internal Coordinate Hamiltonian Model for Fermi Resonances and Local Modes in Methane. J. Chem. Phys. 1997, 106, $831-845$.

(28) Venuti, E.; Halonen, L.; Della Valle, R. G. High Dimensional Anharmonic Potential Energy Surfaces: The Case of Methane. J. Chem. Phys. 1999, 110, 7339-7347.

(29) Wang, X. G.; Sibert, E. L. A Nine-Dimensional Perturbative Treatment of the Vibrations of Methane and its Isotopomers. J. Chem. Phys. 1999, 111, 4510-4522.

(30) Nave, S.; Tiwari, A. K.; Jackson, B. Methane Dissociation and Adsorption on $\mathrm{Ni}(111), \mathrm{Pt}(111), \mathrm{Ni}(100), \mathrm{Pt}(100)$, and $\mathrm{Pt}(110)-(1 \times$ 2): Energetic Study. J. Chem. Phys. 2010, 132, 054705.

(31) Bechtel, H. A.; Kim, Z. H.; Camden, J. P.; Zare, R. N. Bond and Mode Selectivity in the Reaction of Atomic Chlorine with Vibrationally Excited $\mathrm{CH}_{2} \mathrm{D}_{2}$. J. Chem. Phys. 2004, 120, 791-799.

(32) Jiang, B.; Guo, H. Mode and Bond Selectivities in Methane Dissociative Chemisorption: Quasi-Classical Trajectory Studies on Twelve-Dimensional Potential Energy Surface. J. Phys. Chem. C 2013, 117, 16127-16135. 